\title{
Distúrbios da Atenção na Infância e Adolescência
}

\author{
Luiz Celso Pereira Vilanova*
}

\section{RESUMO}

A Sindrome do Déficit de Atençāo na infância e na adolescẻncia è um quadro relativarnente freqüente que ainda é motivo de controvérsias quanto à sua natureza etiopatogênica,seu diagnóstico e sua terapêutica.Este artigo aborda as caracteristicas clinicas e as possibilidades terapêuticas.

\section{UNITERMOS}

Sindrome do Déficit de Atenção-Características clinicas e tratamento.
Prol.-Adjunto; Doutor Chele do Setor de Neurologia Infantil da Disciplina de Neurologia da Escola Paulista de Medicina.
A Síndrome do Déficit de Atenção na infância e na adolescência é um quadro relativamente freqüente, mas que ainda é motivo de controvérsias quanto a sua natureza etiopatogênica, seu diagnóstico e sua terapêutica.

Desde as primeiras descriçōes as características clínicas, os critérios diagnósticos e o próprio nome da síndrome têm sofrido muitas modificações com o passar dos anos ${ }^{(6,10,14)}$. Entretanto, é inconteste que existe um grupo de indivíduos, independentemente do rótulo empregado, que já nos primeiros anos da vida apresenta uma dificuldade maior em manter o foco de seu interesse em uma determinada atividade, mesmo as de natureza lúdica. Na Pré-escola ou na fase escolar esta característica é mais evidente, pois a criança não consegue prestar atenção no que é ensinado, se distraindo com qualquer tipo de estímulo ambiental. Associado a este quadro, outras manifestações podem estar presentes tais como uma inabilidade motora, sendo, muitas vezes, rotulados pelos pais de desajeitados ou desastrados. Além disso, podem apresentar um desenvolvimento de fala mais tardio e lento que as demais crianças. A hiperatividade, que se manifesta por uma tendência de estar sempre se movimentando, constitui num dos sinais clínicos mais frequientes e exulberantes associados ào Síndrome do Déficit de Atenção. Alteração da sociabilidade, labilidade emocional e uma dificuldade de ter seus limites bem definidos são outras características associadas às crianças e adolescentes com Síndrome do Deficit de Atenção ${ }^{(3,6,11,13)}$. Devido à esta plêiade de manifestações clínicas, que podem estar associadas em maior ou menor grau de intensidade, muitos rótulos têm sido utilizados para designar estes indivíduos tais como: lesão cerebral mínima, Disfunção cerebral mínima, distúrbios de aprendizagem e e' ultimamente, síndrome do deficit de atenção com ou sem hiperatividade ${ }^{(4,6,0,11,14)}$ '.

\section{Quadro Clínico}

Apesar de serem crianças habitualmente com inteligência na média ou superior a média(6), apresentam características clínicas que denotam, logo nos primeiros anos de vida, alterações no seu processo de desenvolvimento neurológico e emocional. As etapas do desenvolvimento, no primeiro ano de vida, geralmente se estabelecem dentro dos parâmetros da normalidade, sem chamar a atenção dos pais. Entretanto, alguns pacientes, já nesta fase precoce do desenvolvimento, mostram-se mais irritadiços, chorandó muito nos primeiros meses de vida, movimentando-se muito durante o sono e acordando várias vezes durante a noite. A partir do primeiro aniversário, os pais começam perceber mais nitidamente que a criança é mais 
agitada , necessitando constantemente ser vigiada, pois senão provoca alguma "arte", quebrando com freqüência os seus brinquedos, perdendo rapidamente o interesse por brinquedos ou situações, procurando sempre novos estímulos. Muitas das crianças com Síndrome do Déficit de Atenção, especialmente os meninos, apresentam um desenvolvimento da fala mais lento e uma imprecisão fonoarticulatória que se manifesta através de trocas, omissões e distorções fonêmicas ${ }^{(6,17)}$. Esta alteração da produção fonêmica pode estar associada também a uma alteração do ritmo da fala, especialmente um ritmo mais acelerado, , caracterizando uma taquilalia. Este distúrbio de fala quando não diagnosticado e tratado o mais precoce possível, poderá, mais tarde, acarretar dificuldade ou mesmo alteração no processo de alfabetização da criança.

Além do distúrbio da fala, muitas das crianças com Síndrome do Déficit de Atenção apresentam também uma coordenação motora inadequada ${ }^{(6,11)}$. Apesar de terem os automatismos adquiridos nos primeiros meses de vida iguais aos das demais crianças, tais como sentar e andar, os automatismos adquiridos mais tardiamente geralmente são adquiridos de modo mais lento e são realizados com maior imprecisão. Deste modo, os atos práxicos, especialmente os que envolvem a utilização de instrumentos tais como amarrar um sapato ou utilizar o lápis, só são adquiridos após muito treino e, mesmo assim, a qualidade com que é realizada deixa sempre a desejar em relação às demais crianças ${ }^{(13)}$. Associada à esta inabilidade motora, estas crianças apresentam também um desenvolvimento da noção têmporo-espacial inadequada que pode ser evidenciada através de seus desenhos ou da incapacidade de reconhecer símbolos gráficos semelhantes entre $\mathrm{si}$, mas que se diferenciam apenas pela sua disposição espacial( tais como o número 3 e a letra $E$ ou as letras b, p, q, d. $)^{(15)}$.

Estas funções que nas demais crianças são adquiridas quase naturalmente, apenas com a possibilidade de experimentar $\mathrm{e}$ vivenciar, nas crianças com Síndrome do Deficit de Atenção são difícieis de serem adquiridas, levando a um desgaste emocional da própria criança e de seus familiares, especialmente quando a hiperatividade está presente, pois os familiares e os professores passam a maior parte do tempo reprimindo, chamando a atenção da criança para não fazer isto ou aquilo ou para parar quieto por certo tempo. . Os problemas na área afetivo-emocional, associados ao insucesso escolar podem colaborar para um futuro desajuste social e, conseqüentemente, a própria deliquência juvenil.

\section{ASPECTOS FISIOPATOLOGICOS}

O substrato anatômico da ATENÇÃO é formado por várias regiões cerebrais que participam no controle desta função. Desde os trabalhos de MORUZZI e MAGOUN em 1949 ${ }^{(16)}$ tem-se demonstrado a participação da Formação Reticular, uma das estruturas que compõe o denominado SISTEMA ATIVADOR ASCENDENTE. Este SISTEMA tem a função de ativar outras áreas cerebrais ${ }^{(5)}$, influenciando áreas relacionadas com a MEMÓRIA e a própria MOTIVACÃO do indivíduo. Por outro lado, a capacidade de variar o foco de Atençāo e de mantê-la em tarefas monótonas se encontra diretamente vinculada à função da Região PRÉ-FRONTAL ${ }^{(13)}$. Esta região , que por sua vez pode ser ativada pela Formação Reticular , ativa outras áreas cerebrais , inclusive as que tem função relacionada ao controle da noção Espacial e da Linguagem, o que poderia justificar o porquê destas crianças apresentarem manifestações clínicas tão ricas como já salientado. Entretanto , os esporádicos estudos iniciais realizados com abordagem anátomo-patológica não evidenciaram alterações lesionais nestas regiões cerebrais, sendo, para alguns autores, até um ponto importante na sustentação do diagnóstico da Síndrome do Déficit de Atenção ${ }^{(1)}$. Posteriormente, os autores ${ }^{(1,18)}$, através de estudos com neurotransmissores e neuromoduladores, procuraram identificar a alteração neurológica, mas sempre com as devidas limitações em trabalhos com seres humanos. Em resumo, estas pesquisas têm encontrado indícios de um estado HIPODOPAMINÉRGICO resultante ou de um déficit na ressíntese da Dopamina ou de uma menor sensibilidade pós-sináptica. ${ }^{(1,18)}$. Por outro lado, existem vários trabalhos que procuraram encontrar e caracterizar alterações que poderiam ser quantificadas do ponto de vista neurofisiológico. Assim, SHOUSE e LUBAR ${ }^{(19)}$ encontraram uma hipocondução electrodérmica. CONTE e KINSBOURNE ${ }^{(7)}$ acreditam que existam dois padrōes de indivíduos com Síndrome do Déficit de Atenção: atividade electrodérmica espontânea estável e atividade electrodérmica lábil. Segundo estes autores, seria possível predizer, inclusive, a resposta terapêutica com estimulantes nestes pacientes. Por outro lado, PORGES ${ }^{(20)}$ verificou que algumas crianças com Síndrome do Déficit de Atenção apresentavam um controle vagal cardíaco deficiente em atividades que requeriam atenção. Estudos eletrencefalográficos, utilizando análise espectral, têm comprovado estudos anteriores sem esta tecnologia que se demonstra nestas crianças, um excesso de ondas lentas no traçado eletrencefalográfico que tende a diminuir na adolescência ${ }^{(1,13)}$. LOU e colaboradores $^{(9)}$ usando Pet-Scan com Xenon 133 encontraram, nas crianças com Síndrome do Déficit de Atenção hiperativas, uma hipoperfusão nas regiões Estriatais e uma hiperperfusão na região Cortical sensitiva primária. Este mesmo autor assegura que o uso do Metilfenidato reverteu esta tendência. Em algumas crianças, diagnosticadas como Síndrome do Déficit de Atenção, temos encontrado evidências de alteração do Processamento Auditivo Central através da pesquisa de Escuta Dic6tica com estímulo Consoante-Vogal.

\section{DIAGNÓSTICO E TRATAMENTO}

$\mathrm{Na}$ Síndrome do Déficit de Atenção, por se tratar de um quadro sindrômico, é importante estabelecermos o diagnóstico etiológico, assim como identificarmos os fatores agravantes ou 
desencadeantes para podermos estabelecer um planejamento mais adequado do tratamento. $\mathrm{Na}$ atualidade, o diagnóstico é eminentemente clínico, baseado nos dados colhidos numa anamnese minusiosa, pela observação clínica do paciente, pelo exame neuropediátrico e pelas avaliações e testagens das áreas comprometidas, já que estes dados serão fundamentais no planejamento do tratamento do paciente. $O$ tratamento medicamentoso deve, a meu ver, ser sempre um coadjuvante do tratamento do indivíduo, devendo ser utilizado com parcimônia e por tempo não muito prolongado. Apesar de não existir um consenso, o emprego de estimulantes do Sistema Nervoso Central pode melhorar significativamente o Distúrbio de Atenção e a Hiperatividade, tendo pouca ou nenhuma ação nas demais manifestações clínicas. Apesar de sua ação ser apenas sintomática, o tratamento medicamentoso pode contribuir na normalização do comportamento do indivíduo enquanto outras formas de atuação terapêutica são utilizadas. Dos estimulantes mais usualmente empregados ${ }^{(3,4,23)}$, especialmente nos Estados Unidos, o Metilfenidato (RITALINA*)tem sido considerado um dos de melhor ação terapêutica. A dose empregada é em torno de $0,3-1,0 \mathrm{mg} / \mathrm{kg} / \mathrm{dia}$, com dose preferencial na parte da manhã para não interferir com o sono. Uma outra medicação utilizada é o PEMOLINE(CYLERT*), usado no REINO UNIDO há mais de 25 anos e somente, mais recentemente, nos ESTADOS UNIDOS. Entretanto, existem raras publicações ${ }^{(2,}$ $21,22)$, que relatam efeitos colaterais importantes tais como a hepatotoxidade ou tics atribuídos ao seu uso, apesar de serem raros outros efeitos colaterais. A imipramina (TOFRANIL*)também tem sido utilizada como uma boa alternativa para o tratamento medicamentoso, especialmente no nosso meio, por ser mais fácil de ser adquirido pelos pacientes. A Cafeína, muito empregada no passado nestes pacientes, em doses de $100-300 \mathrm{mg} / \mathrm{dia}$, talvez tenha um efeito mais de placebo do que real. Se por um lado não nos parece adequado não auxiliar estas crianças, seus pais e professores a terem um comportamento mais adequado, menos hiperativo, mais atento sem ser necessário um esforço sobrenatural, através do uso de medicamentos, por outro lado a medicação pode ter efeitos colaterais. Os efeitos colaterais mais comuns(4), especialmente com o uso do Metilfenidato, são a diminuição do apetite sendo necessária a monitorização do peso, insônia, mal estar abdominal, labilidade emocional, sedação, diminuição do ritmo do crescimento e a precipitação de tics devendo ser contraindicado em pacientes portadores de tics, ou filhos de pais de tics complexos. Quanto ao receio que o uso destas medicações facilitem, num futuro à droga habituação, os estudos não têm comprovado este receio, ao contrário, estes jovens adequadamente tratados, tendem a ser menos deliquientes $(3,23)$. $\mathrm{O}$ tratamento não deve se restringir apenas ao uso de medicamentos, mesmo porque a maioria destes pacientes apresenta, como já comentado, um comprometimento mais extenso do que uma alteração da Atenção ou a Hiperatividade. Deste modo, dependendo das manifestações clínicas, pode ser necessário uma terapia fonoaudiológica, terapia corporal, ludoterapia ou uma abordagem psico-pedagógica para aprimorar a sua performace e melhorar a sua conduta.

\section{SUMMARY}

Attention Deficit Disorders are relatively common in childhood and adolescence. However it is still a controversial topic as to its etiopathogenesis, diagnosis and therapy. The aim of this article is to describe the clinical aspects of the disorder and discuss possible therapy.

\section{Bibliografia}

1 POLAINO-LORENTE, A $\theta$ CABANYES, J-Bases neurofisiológicas y aproximacion neuropsicológica al estudio de la hiperactividad infantil. Rev. esp. Fislol. , 45, Supl. :255-264 1989.

2 PRATT, D. S. e DUBOIS, R. S. -Hepatotoxicity due to Pemoline(Cylert):A report of two cases. J. Pediatr. Gastroenterol. Nutr. 10(2):2392411990.

3 WOLRAICH, M. L. et al. -Stimulant medication use by primary care physicians in the treatment of Attention Deficit Hyperactivity Disorder. Pediatrics 86(1):95-101 1990.

4 MENTAL HEALTH COMMITTEE, CANADIAN PAEDIATRIC SOCIETY-Use of methylphenidate for attention deficit hyperactivity disorder. Can Med Assoc. J. 142(8):817-818, 1990.

5 Luria, A. R. -El cerebro en accion 2 ed. Ed Fontanella Barcelona 1979.

6 LEFEVRE, A. B. - Disfunçāo Cerebral Mínima. Sarvier, São Paulo, 1975

7 CONTE, R. E KINBOURNE, M. -Psychophysiology, 25:64-70, 1988.

8 PORGES, S. W. -Ped. Clin. N. Amer. , 31, 371-385, 1984.

9 LOU, H. C. et all-Arch. Neurol., 41, 825-829, 1984.

10 AMERICAN PSYCHIATRIC ASSOCIATION, COMITEE ON NOMENCLATURE and STATISTICS. DIAGNOSTICAL MANUAL OF MENTAL. DISORDERS. 3ed. revised. Washington, DC: American Psychiatric Association; 1987.

11 DIAMENT, A. E CYPEL, S. -Neurologia Infantil Lefevre. 2ed. , Liv. Atheneu, Rio de Janeiro, 1989.

12 SEIDEL, W. T. e JOSCHKO, M. -Evidence of Difficulties in sustained Attention in Children with ADDH Journal of Abnormal Child Psychology. 18(2), 217-229, 1990.

13 GADDES, W. H. -Learning Disabilities and Brain Function-A neuropsychological Approach, New York 1985, 2 ed. Springer-Verlag,

14 CAREY, W. B. -A suggested solution to the confusion in attention deficit diagnosis. Clin. Pediat. , 27:345-349, 1988.

15 DE RENZI, ENNIO-Hemispheric asymmetric as evidence by spatial disorders. in KINSBOURNE, M. -Asymmetric Function of the Brain. -Cambridge University Press, 1978.

16 MORUZZI, G. e MAGOUN, H. W. -Brain stem reticular formation and activation of the EEG. Electroencephalography and clinical neurophysiology. ;1:455-473, 1949.

17 INGRAM, T. T. S. -Speech disorders in childhood. Pediat. Clin N. Amer. 15:611, 1968.

18 RASKIN, L. A. , SHAYWITZ, S. E. ;SHAYWITZ, B. A. ;ANDERSON, G. M. \& COHEN, D. J. -Neurochemical correlates of attention deficit disorder. Pediatric. Clin. N. Amer. , 31:387, 1984.

19 SHOUSE, M. N. E LUBAR, J. F. - Pediatrics, 62:343-351, 1978.

20 PORGES, S. W. -Ped. Clin. N. Amer. 31:371-385, 1984

21 PATTERSON, J. F. -Hepatitis associated with pemoline. South Med. J. , 7:938, 1984.

22 BACHMAN, D. S. -Pemoline induced Tourette's disorder:a case report. Am. J. Psychiatry, 138:1116-7, 1981.

23 HECHTMAN, L. ;WEISS, G \& PERLMAN, T. - Young adult outcome of hiperactive children who received long-term stimulant treatment. J. Am. Acad. Child. Psychiatric, 23:261-269, 1984. 